\title{
Comparison of Early Maladjusted Schemas, Hidden Anxiety, and Cognitive Regulation of Emotion in Normal and Delinquency Juveniles
}

\author{
Faeze Eshaghi Moghaddam Kate Shamshir*, Mohammad Hossein Abdollahi, Mahnaz Shahgholian \\ Department of Psychology, Kharazmi University, Tehran, Iran
}

\section{A BSTRACT}

Introduction: Delinquency is one of the social problems which is usually erupted during adolescence and caused serious problems for them, their families, and the society. There are various factors lead to delinquency, like early maladaptive schemas, anxiety, and cognitive regulation of emotion. This study was aimed to compare the early maladaptive schemas, hidden anxiety, and cognitive regulation of emotion in normal and delinquent juveniles in Mashhad, Iran. Materials and Methods: In this causal-comparative study, there were two groups consisted of 60 male students (aged 12-17 years), divided in normal and delinquent groups. The group of normal juveniles (from high school) was selected by multistage cluster sample and the group of juvenile delinquents (from offenders group in juvenile institution) was selected by simple random sample. The questionnaires were concluded the Young early maladaptive schemas questionnaire, the Spielberger State-Trait Anxiety questionnaire, and the Cognitive Emotion Regulation questionnaire by Garnefski \& Kraaij. Results: Data indicated that the mean scores of early maladaptive schemas in the juvenile delinquents were higher than in normal juveniles. In contrary, the mean scores of hidden anxiety and positive cognitive regulation of emotion were significantly higher in normal group. Regarding the negative cognitive regulation of emotion, there was no difference between two groups. Conclusion: According to significant difference of early maladaptive schemas, hidden anxiety, and positive cognitive regulation of emotion between the delinquent and the normal groups, individual adjustment as well as psychological and social growth can be advantageous and helpful.

\section{Key words:}

1. Juvenile Delinquency

2. Anxiety

3. Emotions

* Corresponding Author: Faeze Eshaghi Moghaddam Kate Shamshir

E-mail: Faeze.eshaghi67@gmail.com 


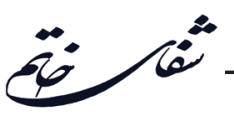

مقايسُٔ طر حوارههاى ناكار آمد اوليه، اضطراب پننهان و تنظيم شناختى هيجان در نوجوانان بهنجار و بزهكار

فائزه اسحقى مقدم كته شمشير"، محمد حسين عبداللهى، مهناز شاهقليان

كروه روانشناسى عمومى، دانشَاه خوارزمى، تهران، ايران

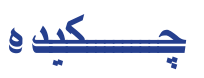

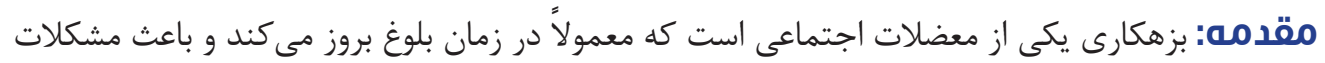

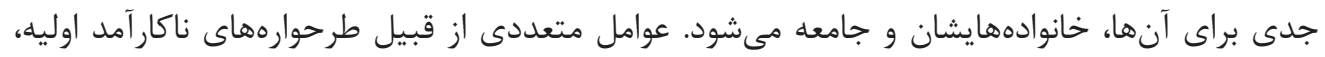

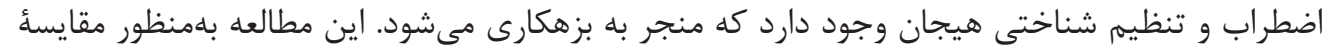

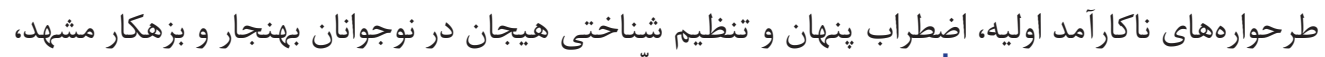

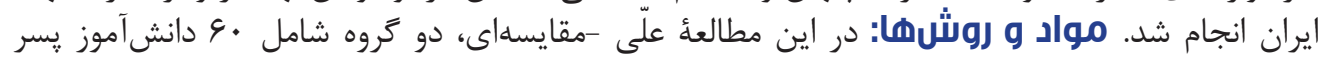

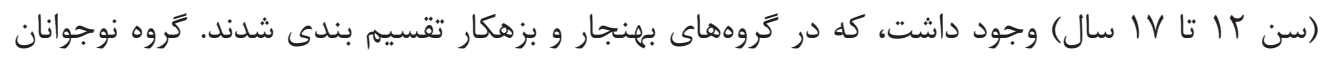

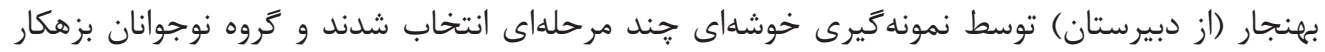

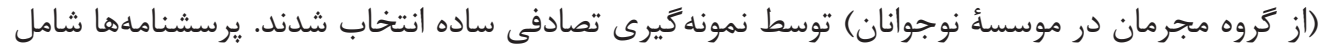

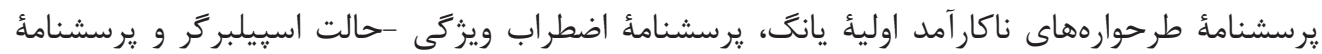

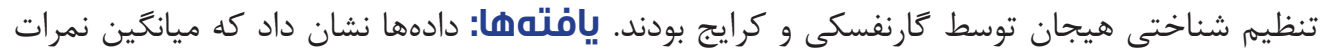

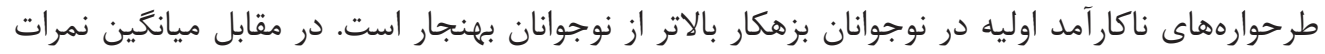

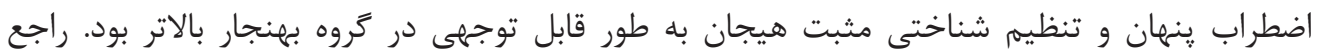

كليد وازهها:

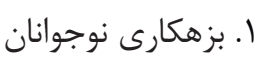

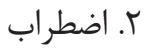

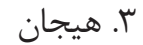

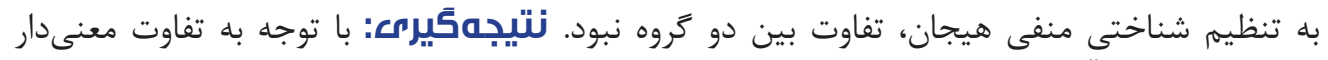

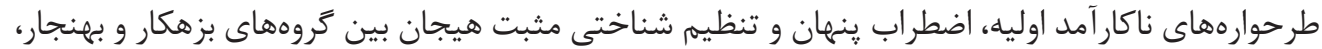

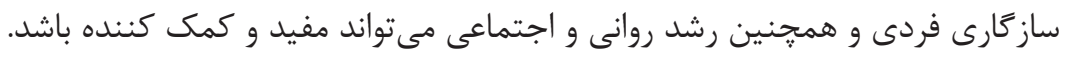

* نويسنده مسئول: فائزه اسحقى مقدم كته شمشير

آدرس الكترونيكى: Faeze.eshaghi67@gmail.com 


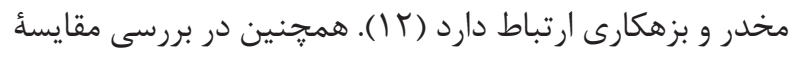

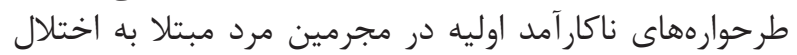

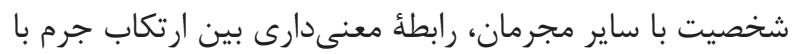

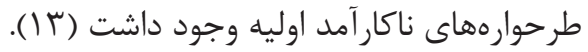
از ييامدهاى وجود طرحوارههاى ناكارآمد اوليه، تداوم اختلال

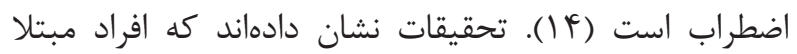

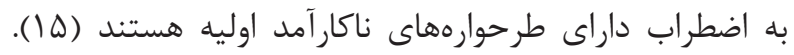

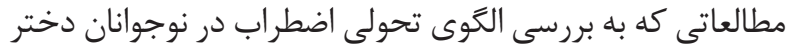

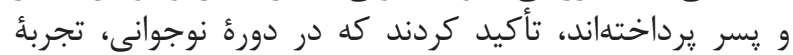

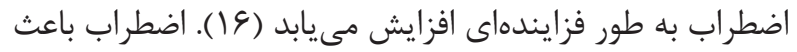

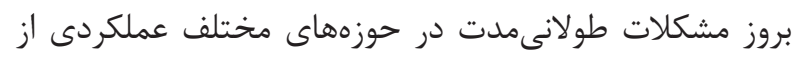

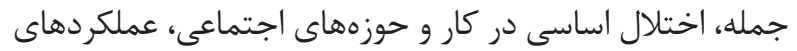

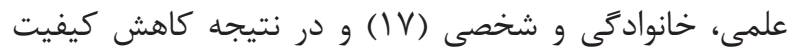

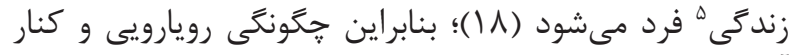

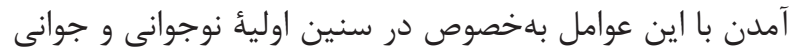

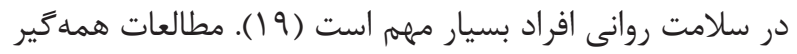

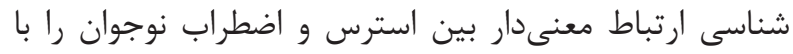

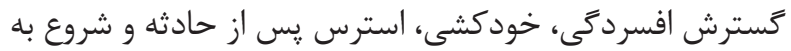

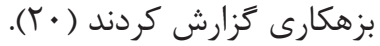

در برخى مطالعات، اضطراب به دو نوع اضطراب آشكار ؤ اضطراب

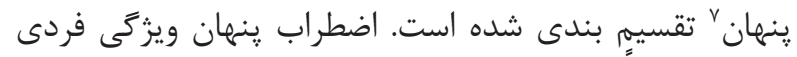

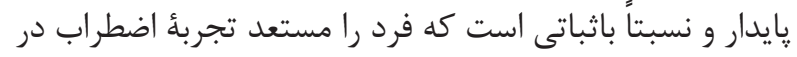

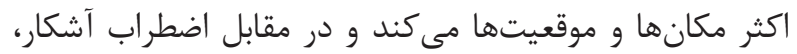

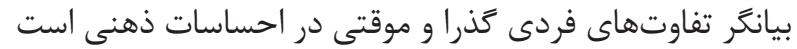

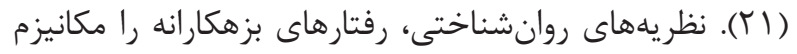

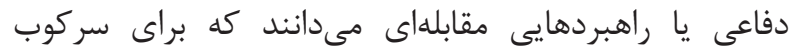

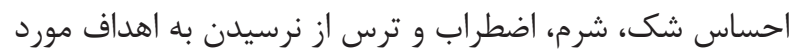

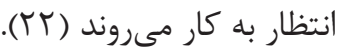

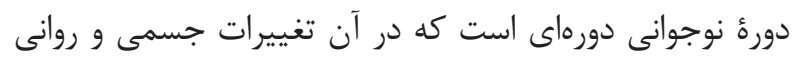

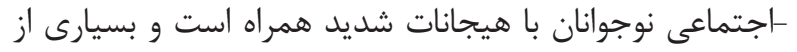

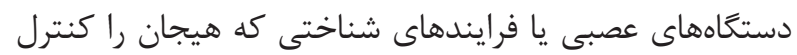

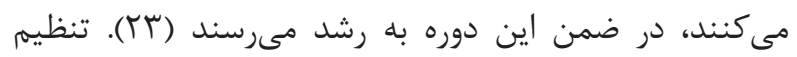

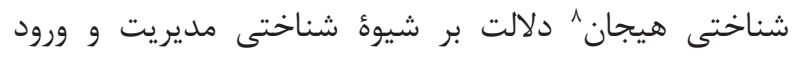

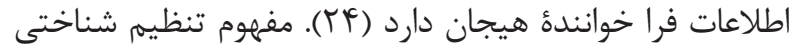

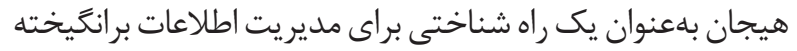

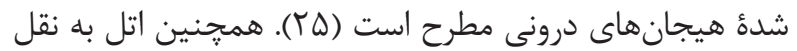

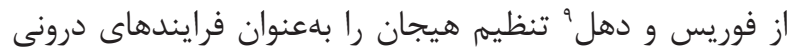

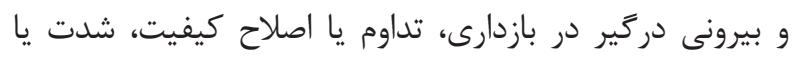

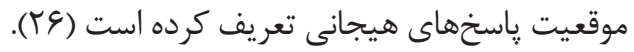
بررسى متون و مطالعات روانشناختى نشان مىدهيد كه تنظيم

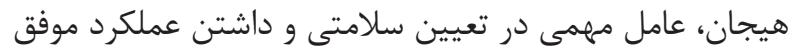

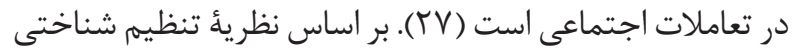

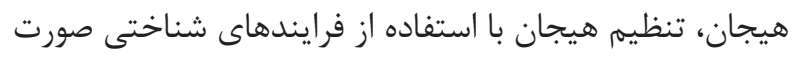

\section{${ }^{1}$ Adolescence}

${ }^{2}$ Delinquency

${ }^{3}$ Schema

${ }^{4}$ Early maladjusted schemas

${ }^{5}$ Quality life

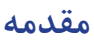

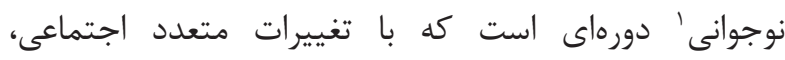

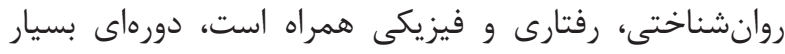

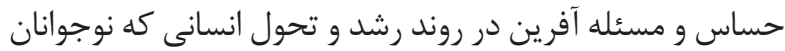

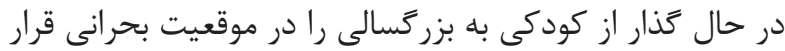

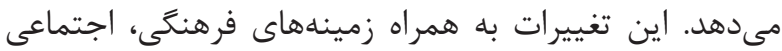

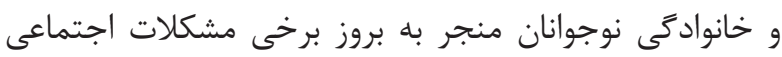

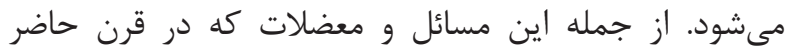

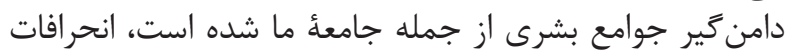

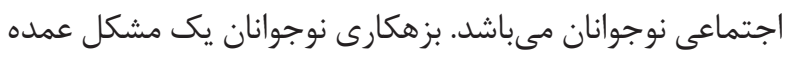

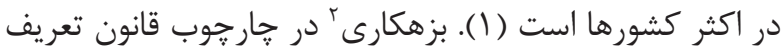

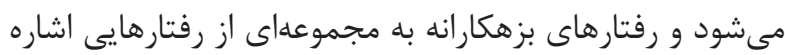

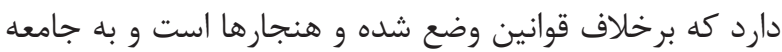

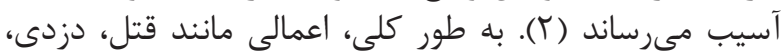

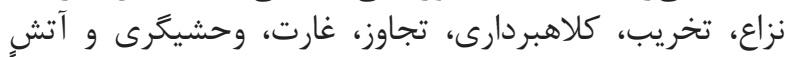

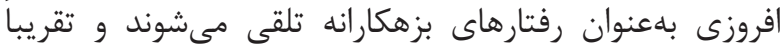
تمامى جوامع در تعريف آن اتفاق نظر دارند (َّ).

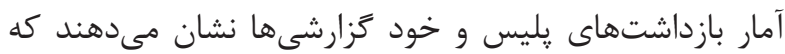

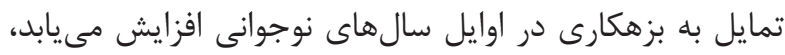

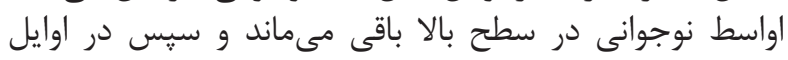

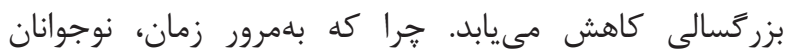

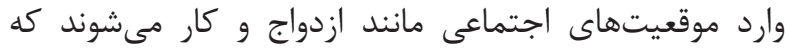

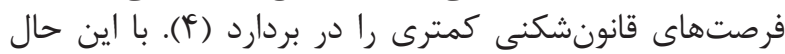

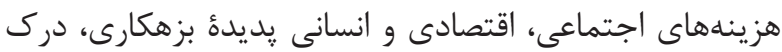

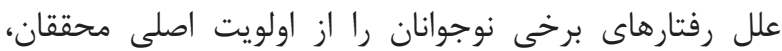

متخصصان و مسئولين ذىربط نرار نوار داده است (ه).

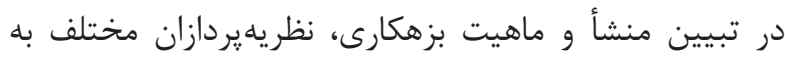

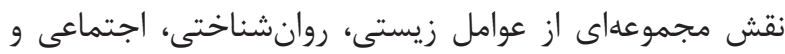

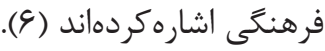

يكى از عوامل مهمى كه امروزه به آن توجه خاصى مبر مبذول

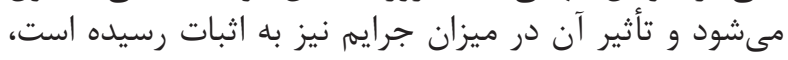

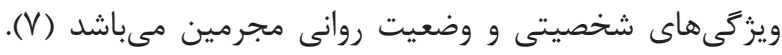

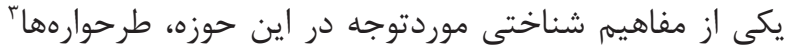

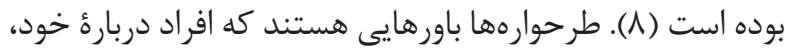

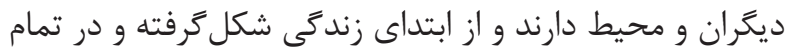

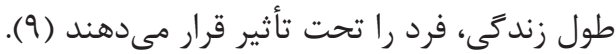

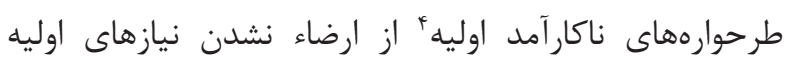

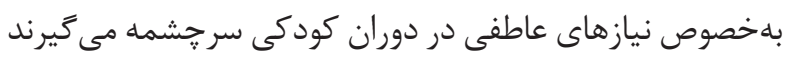

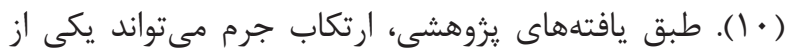

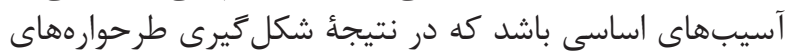

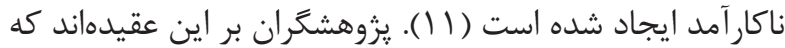

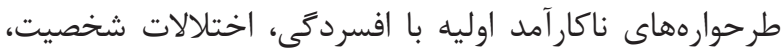

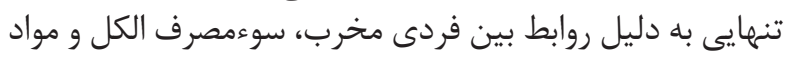

\footnotetext{
${ }^{6}$ Abvious anxiety

${ }^{7}$ Hidden anxiety

${ }^{8}$ Cognitive emotion regulation

${ }^{9}$ Forees \&Dehel
} 


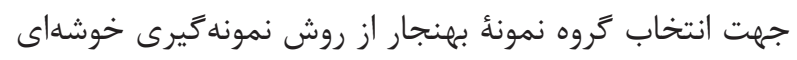

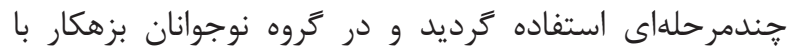

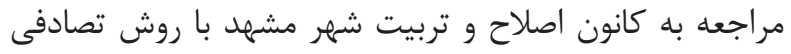

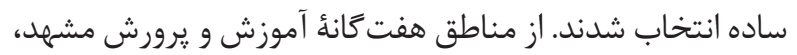

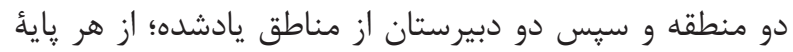

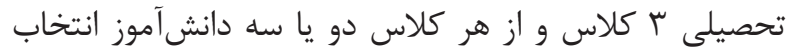

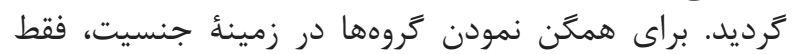

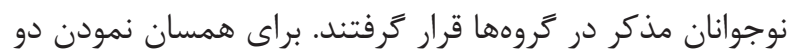

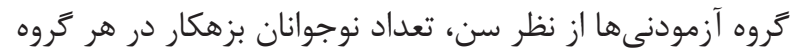

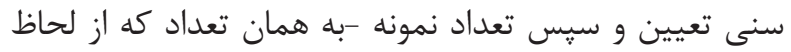

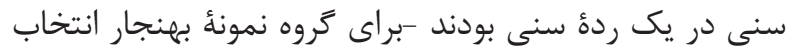

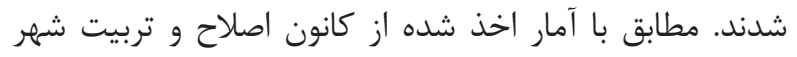

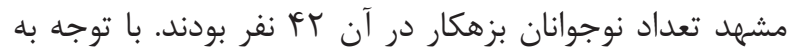

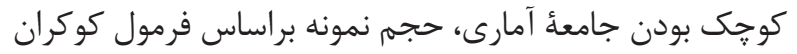

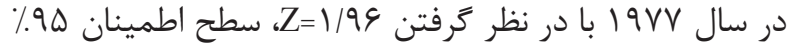

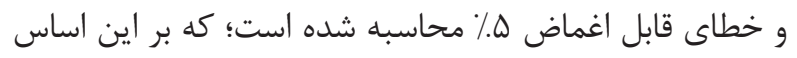

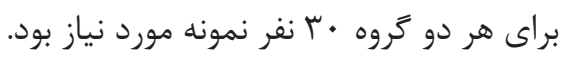

$$
\begin{gathered}
Z_{a / r}=1 / 9 q \\
N=r r \\
p=\cdot / \Delta \\
q=\cdot / \Delta \\
e=.1 .9 \Delta
\end{gathered}
$$$$
n=\frac{N\left(Z_{a / r}\right) \times p \cdot q}{e^{r}(N-1) \times\left(Z_{a / r}\right) \times p \cdot q}
$$$$
\boldsymbol{n}=\frac{\operatorname{Rr}(1 / 99) \times(\cdot / \Delta \times \cdot / \Delta)}{\boldsymbol{e}^{r}(4 r-1) \times(1 / 99) \times(\cdot / \Delta \times \cdot / \Delta)}=r \cdot / 9 \Lambda
$$

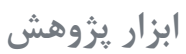

در اين يزوهش از سه ابزار سنجش استفاده شد؛ كه ويزگى هاى روانسنجى آنها به قرار زير است.

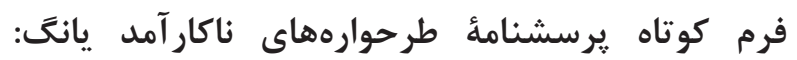

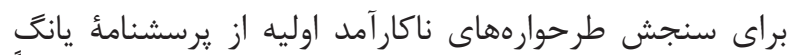

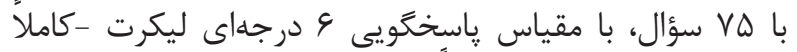

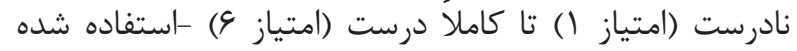

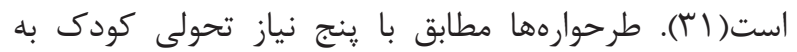

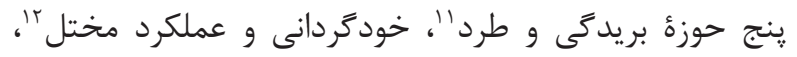

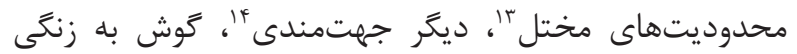

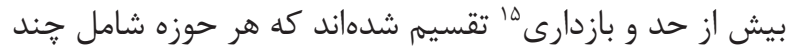

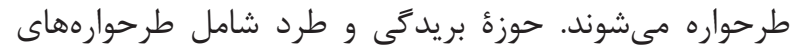

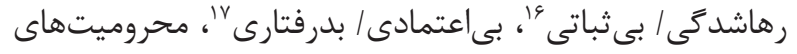

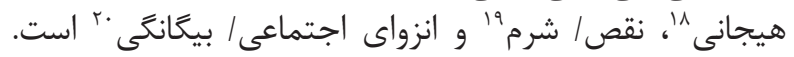

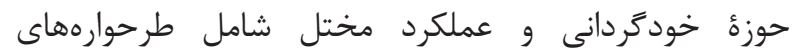

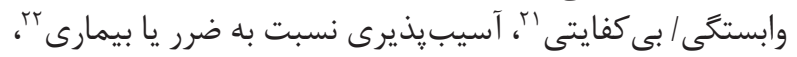

\footnotetext{
${ }^{10}$ Ex post factor

${ }^{11}$ Disconnection\& rejection

${ }^{12}$ Impaired autonomous \& performance

${ }^{13}$ Impaired limits

${ }^{14}$ Other-directedness

${ }^{15}$ Over vigilance /inhibition

${ }^{16}$ Abandonment/instability
}

مى ميرد؛ بهعبارت ديكر فرايندهاى شناختى به افراد كمك

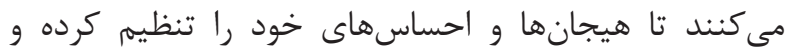

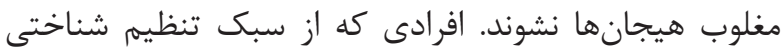
ضعيف استفاده مى كنند، در برابر مشكلات هيجانى آسيبيذ إنذير

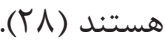

سامانى و صادقى در مطالعة خود راهبردهاى تنظيم شناختى رانى

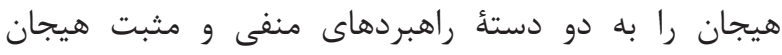

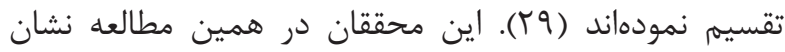

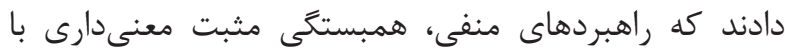

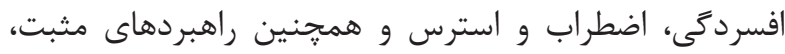

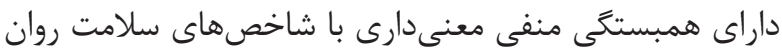

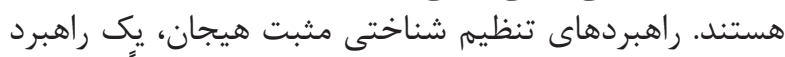

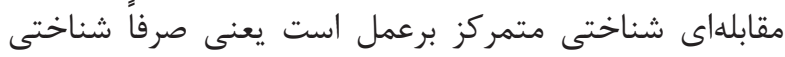

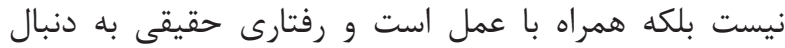

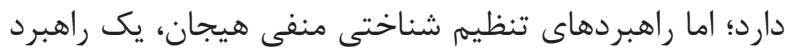

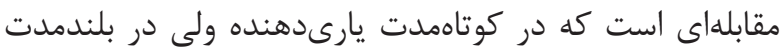

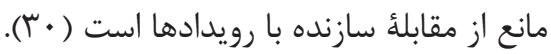

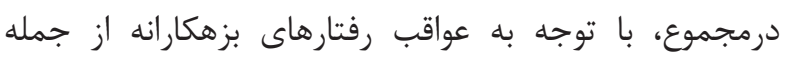

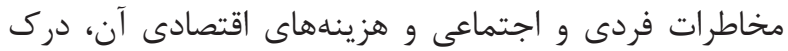

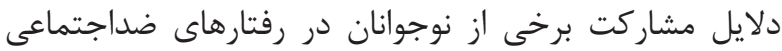

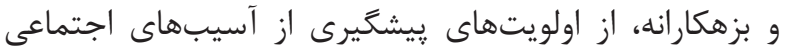

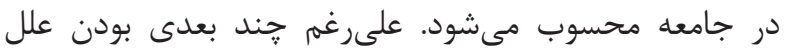

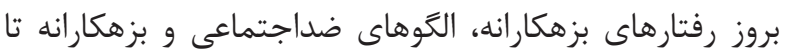

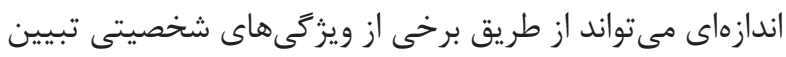

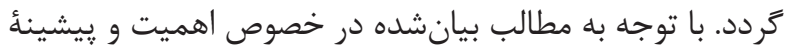

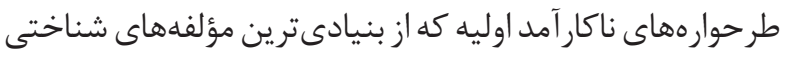

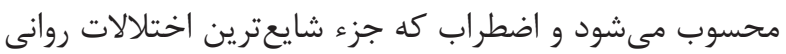

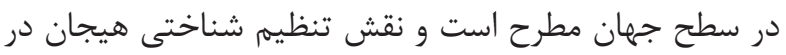

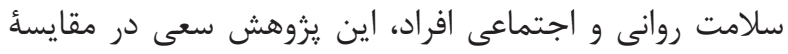

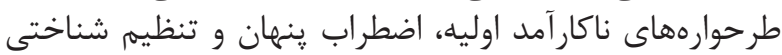
هيجان در نوجوانان بهنجار و بزهكار شهر مشهراب إنه را دارد.

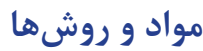

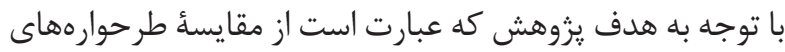

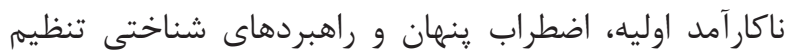

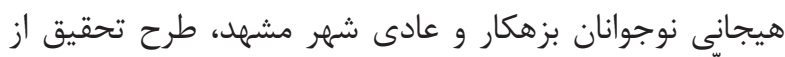

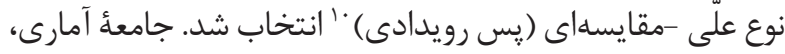

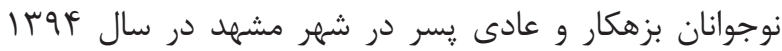

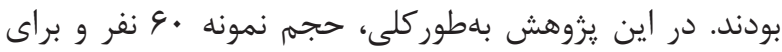

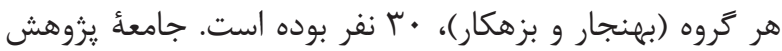

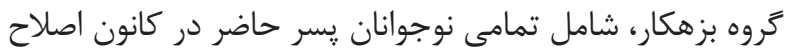

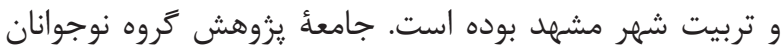

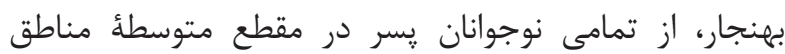
هفت كانئ آموزش و يرورش نهران شهر مشهد انتخاب شده است.

\footnotetext{
${ }^{17}$ Mistrust/abuse

${ }^{18}$ Emotional deprivation

${ }^{19}$ Defectiveness \& shame

${ }^{20}$ Social isolation/alienation

${ }^{21}$ Dependence/incompetence

${ }^{22}$ Vulnerability to harm or illness
} 


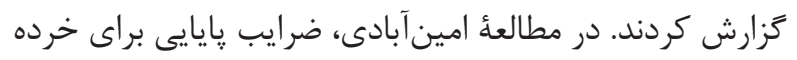

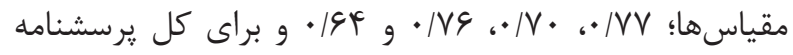

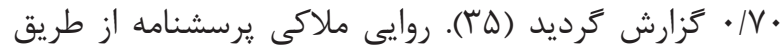

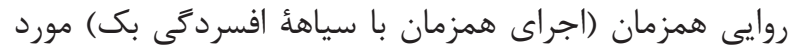
بررسى قراركرفته و روابط درونى خوبى خديى بين خرده مقياسها

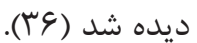

تجزيه و تحليل دادهها

با توجه به اين كه مطالعه از نوع علّى -مقايسهاى است، دادهنها

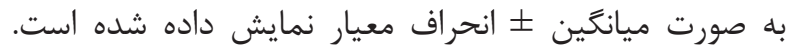

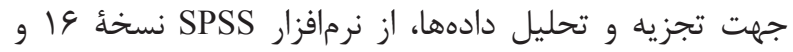

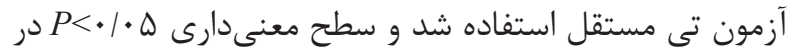
نظر كرفته شد.

بافته ها

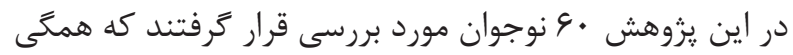

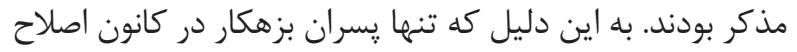

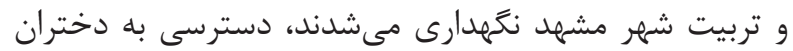

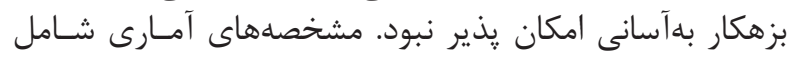

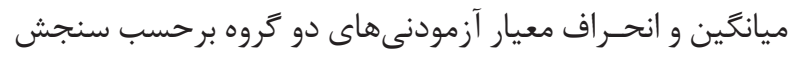

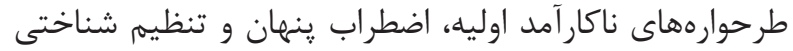

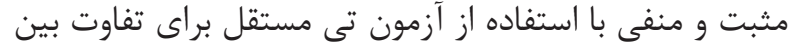

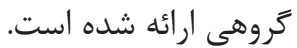

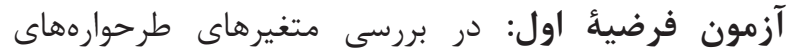

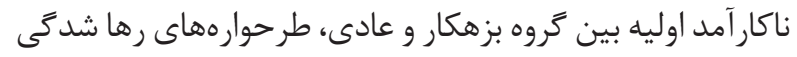

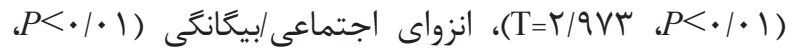

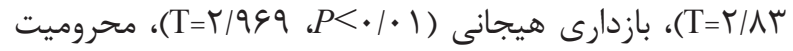

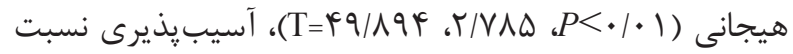

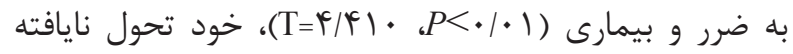
ش

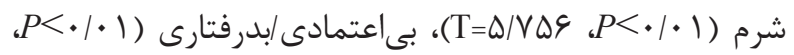

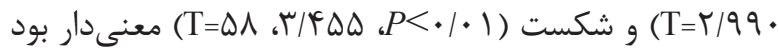

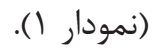

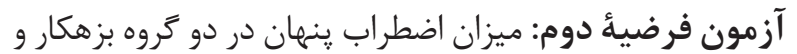

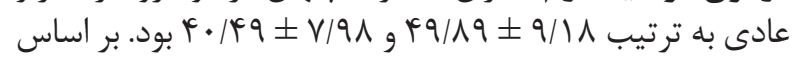

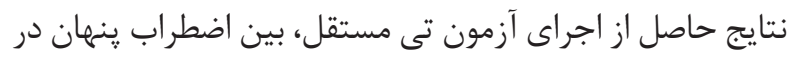

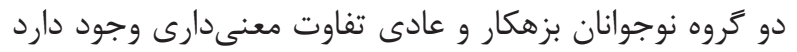

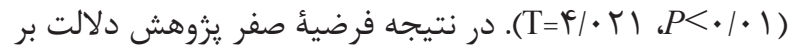
عدم تفاوت معنى دارى بين دو گروه دارد.

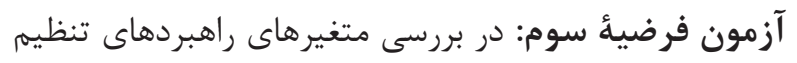

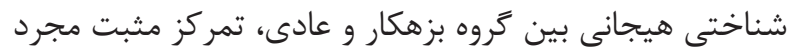

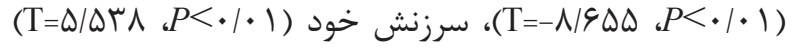

${ }^{23}$ Enmeshment \& undeveloped

${ }^{24}$ Failure

${ }^{25}$ Subjuation

${ }^{26}$ Selfsacrifice

${ }^{27}$ Garnefski

${ }^{28}$ Kraaij

${ }^{29}$ Self-blame

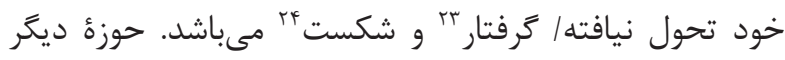

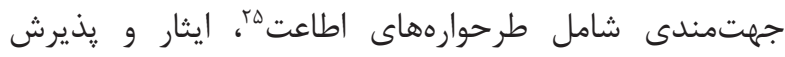

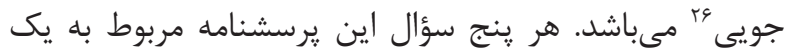

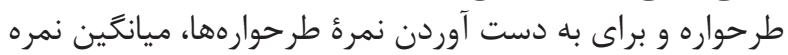

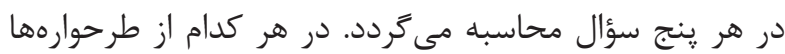

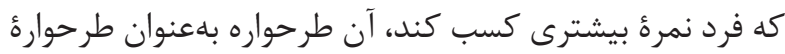

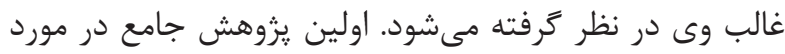

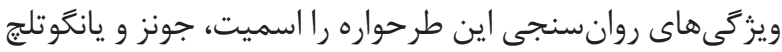

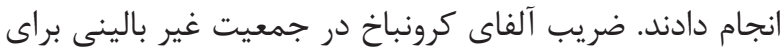

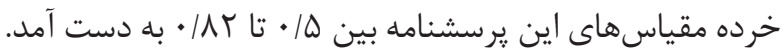

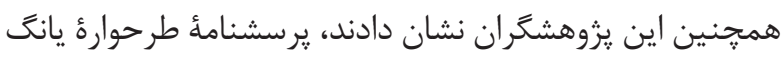

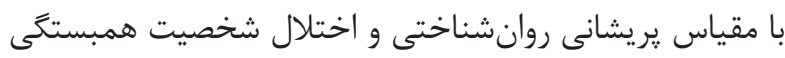

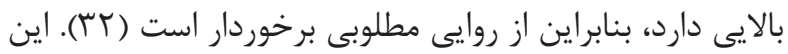

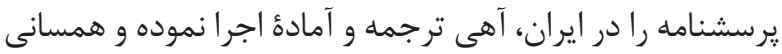

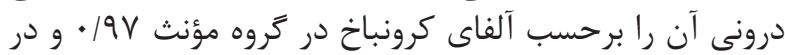

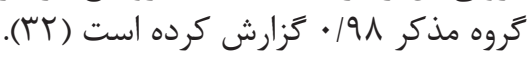

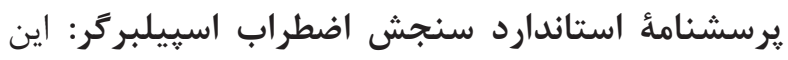

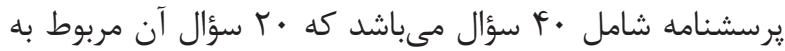

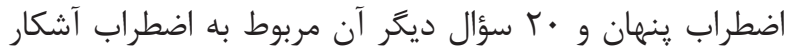

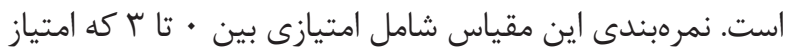

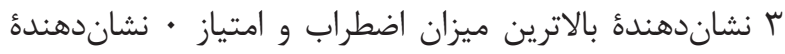

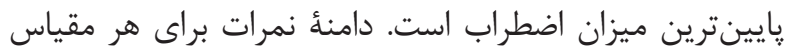

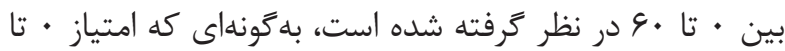

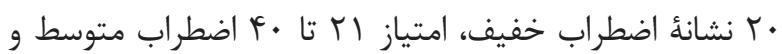

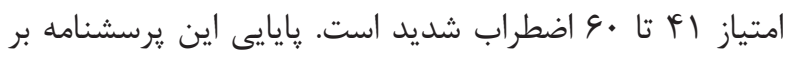

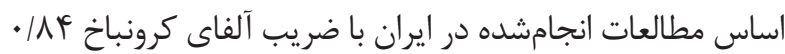

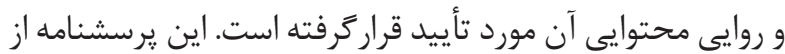

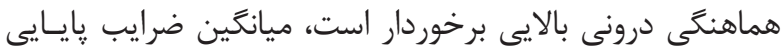

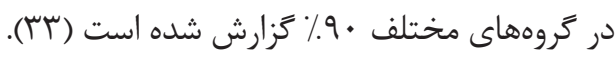

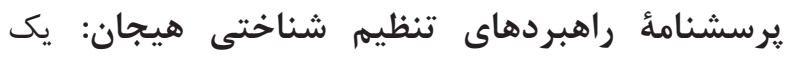

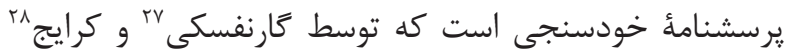

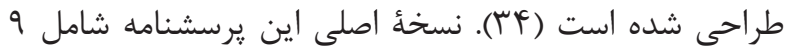

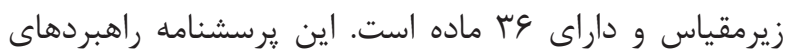

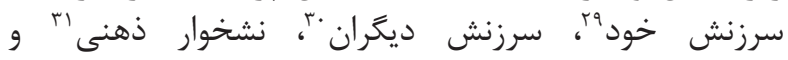

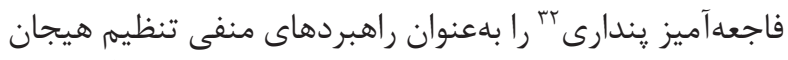

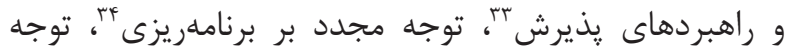

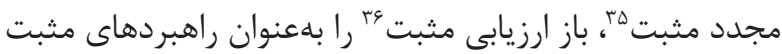

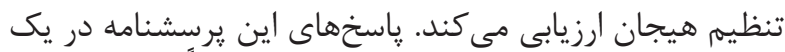

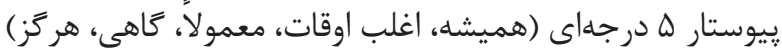

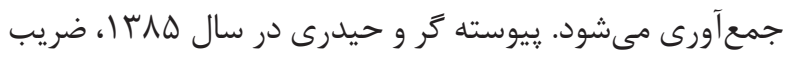

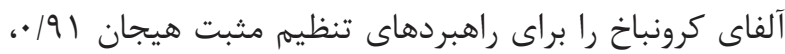

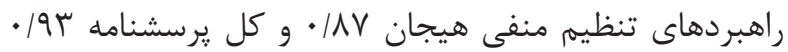

\footnotetext{
${ }^{30}$ Blaming others

${ }^{31}$ Obsessive rumination

${ }^{32}$ Catastrophicing

${ }^{33}$ Acceptance

${ }^{34}$ Refocus on planning

${ }^{35}$ Positive refocusing

${ }^{36}$ Positive appraisal
} 


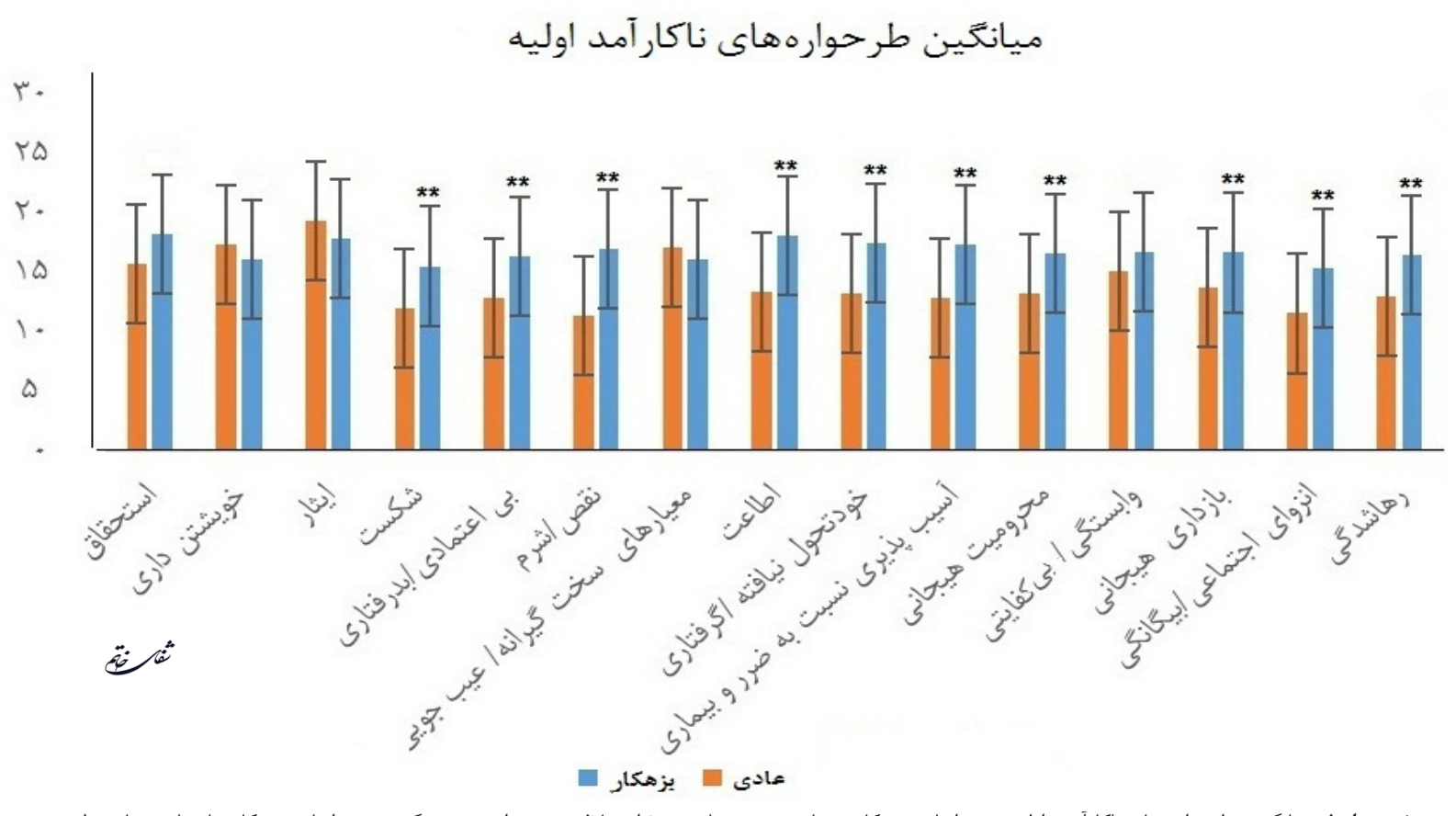

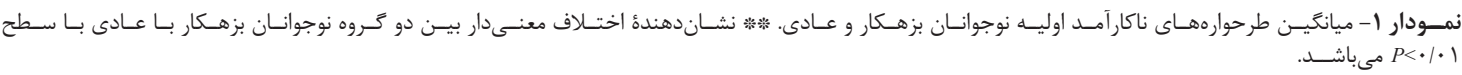

ميانكين راهبردهاى تنظيم شناختى هيجانى

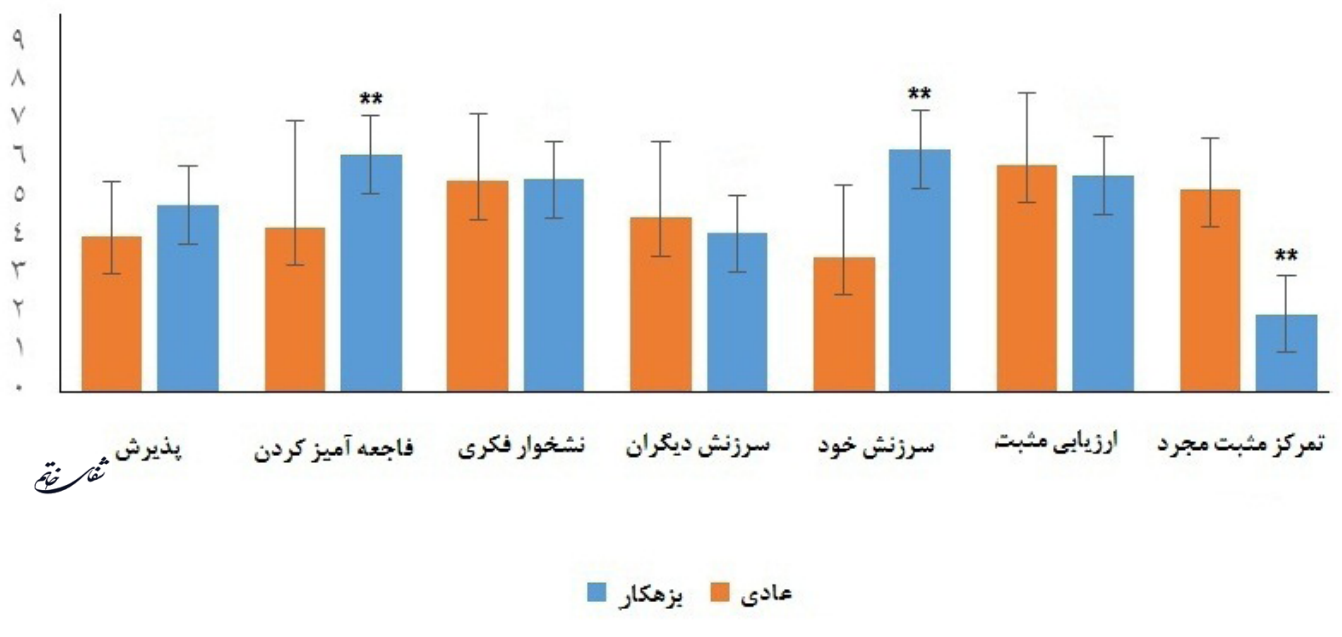

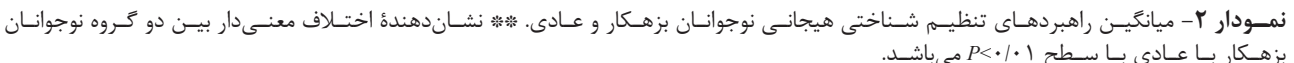

فرضيئ اول: نوجوانان بزهكار، طرحوارههاى ناكارآمد اوليئ

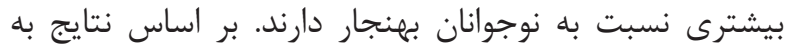

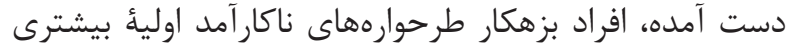

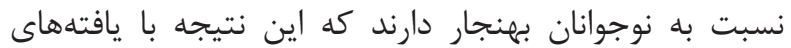

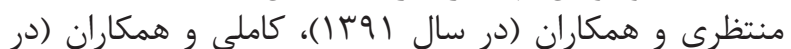

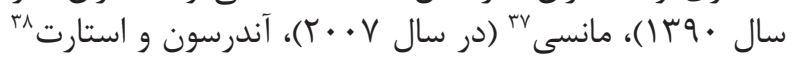

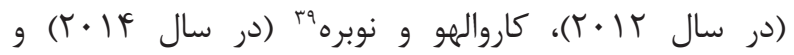

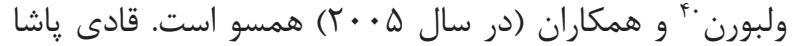
و همكاران در يزوهشى با عنوان بررسى زمينهُ طرحوارههاى

${ }^{38}$ Anderson \& Start

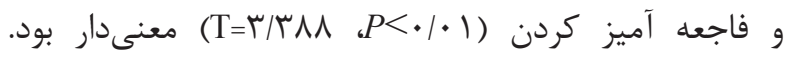

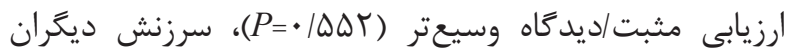

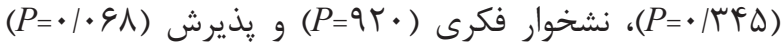
تفاوت معنى دارى وجود ندارد (نمودار ب r).

$$
\text { بحث و نتيجه كيرى }
$$

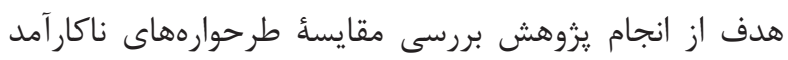

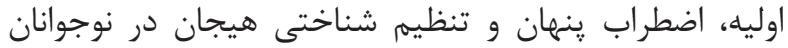
بهنجار و بزهكار شهر مشهد بوده است.

${ }^{39}$ Carvalho \& Nobre

${ }^{40}$ Velborn 
نسبت به نوجوانان بهنجار ضعيفتر عمل مىكنيند. براساس

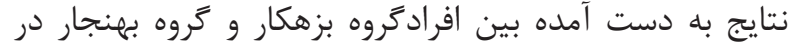

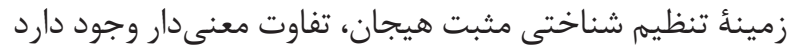

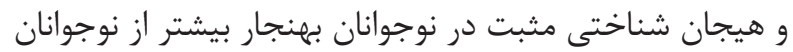

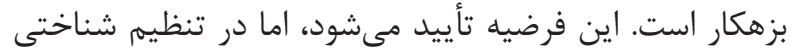

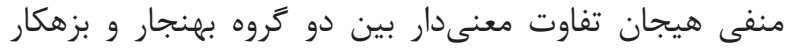

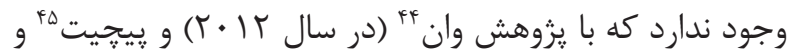

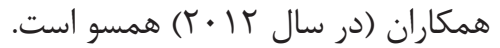

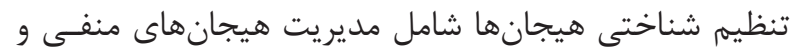

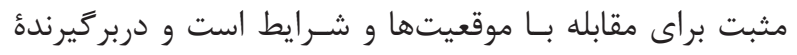

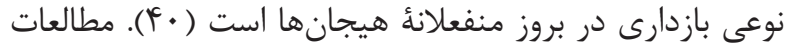

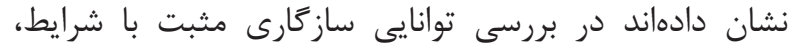

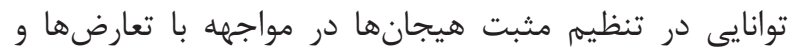

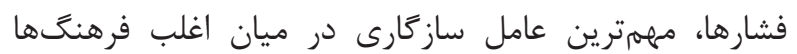

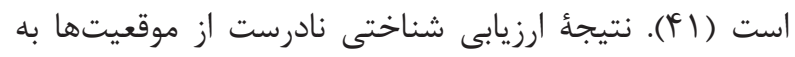

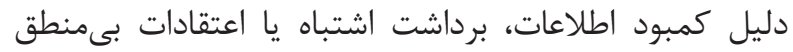

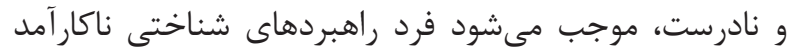

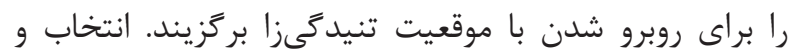

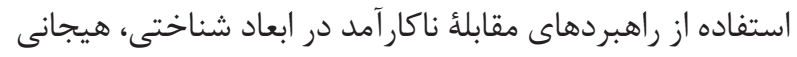

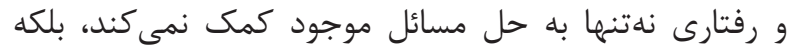

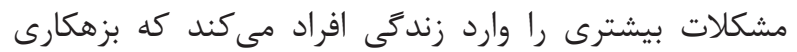
مى تواند يكى از اين مسائل در نوجوانى باشد.

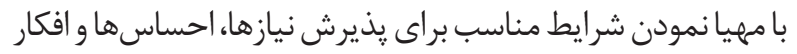

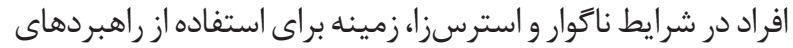

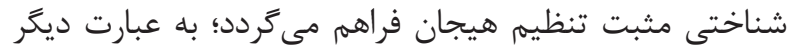

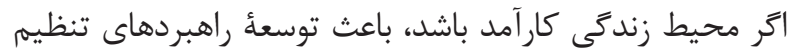

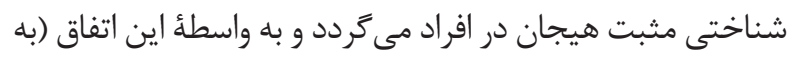

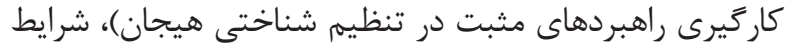

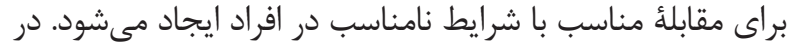

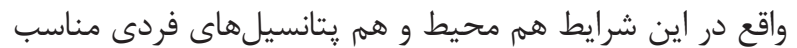

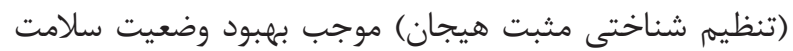

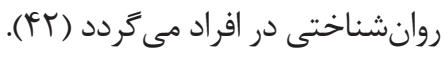

سؤال يزوهش: آيا بين طرحوارههاى ناكارآمد اوليه، اضطراب

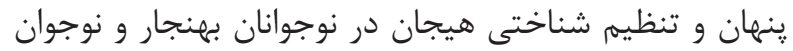

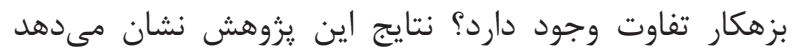

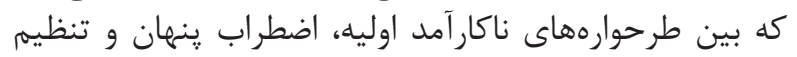

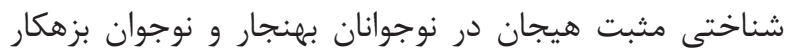

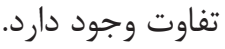

عوامل متعددى در بروز بزهكارى دخيل هستند، بعضى مرتبط

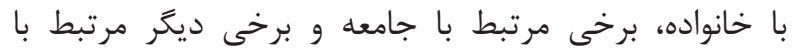

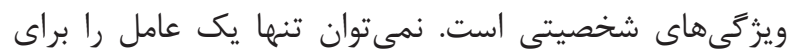

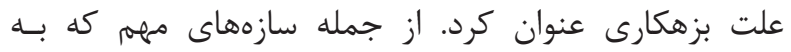

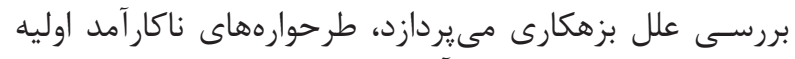

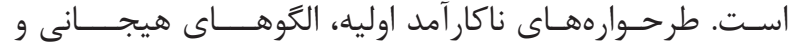

\footnotetext{
${ }^{41}$ Ugurlu

${ }^{42}$ Raj \& Samuel

${ }^{43}$ Jencks
}

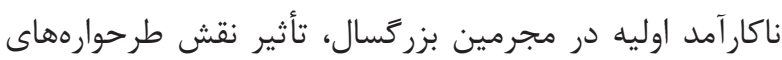

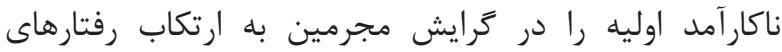

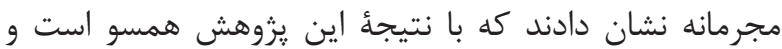
همخوانى دارد و آن را تأييد مى كند. نتئ

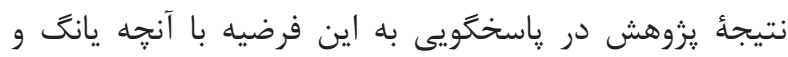

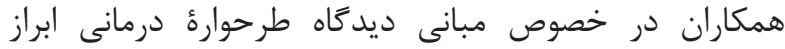

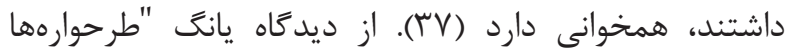

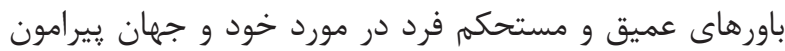

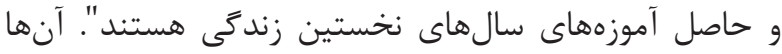

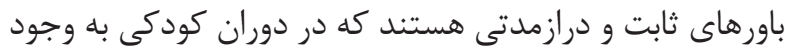

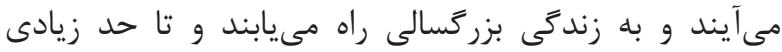

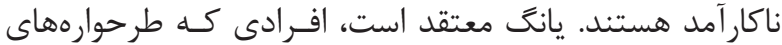

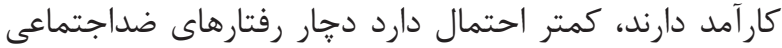

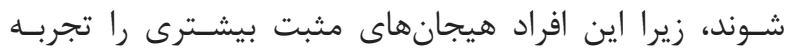

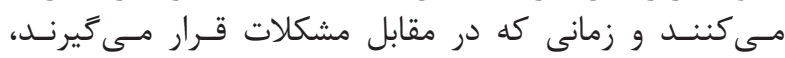

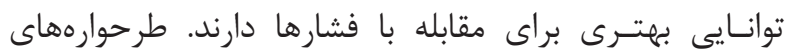

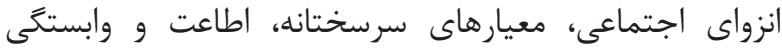

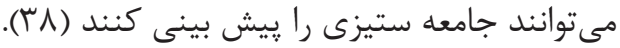
فرضيئ دوم: نوجوانان بزهكار نسبت به نوجوانان بهنجار اضطراب

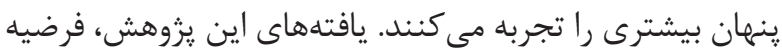

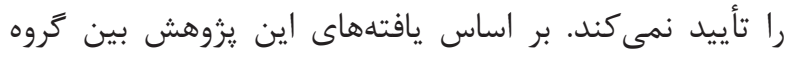

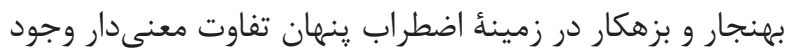

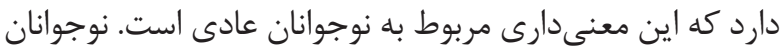

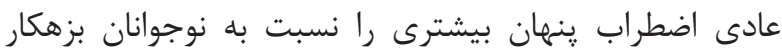

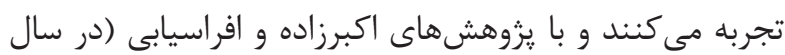

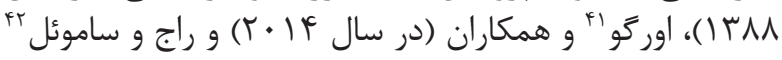

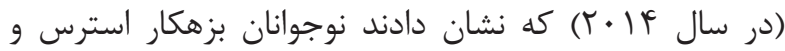

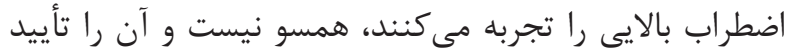

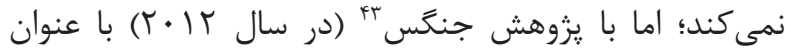

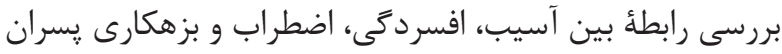
نوجوان همسو است.

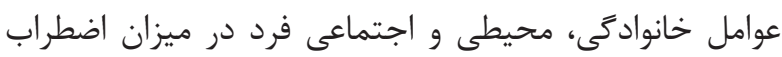

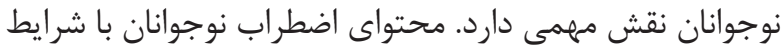

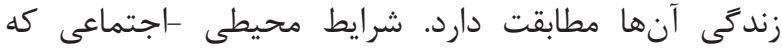

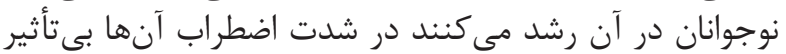

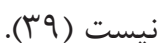

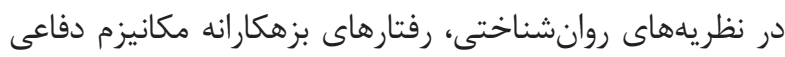

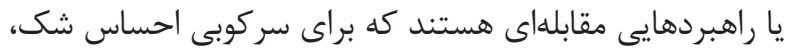

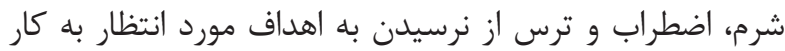

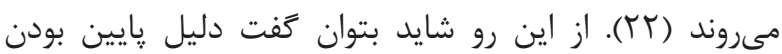

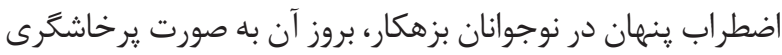

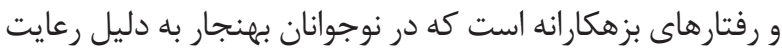

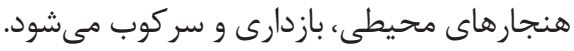
فرضيئ سوم: نوجوانان بزهكار در تنظيم شناختى هيجان ${ }^{44}$ Wan 
سلامت روان، وابسته به عوامل متعددى مىباشد (૧ ؟). به طور كلى و با توجه به يزوهشهاى انجامشده عوامل مؤثر در

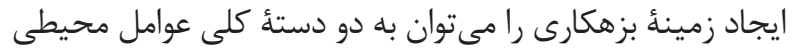

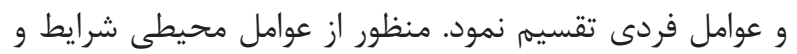

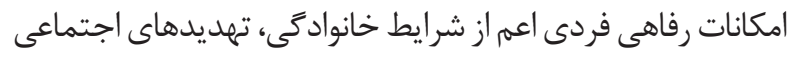

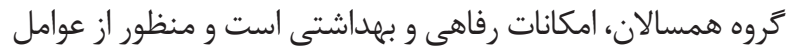

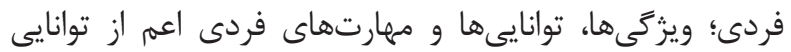

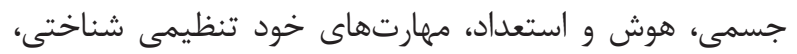
راهبردهاى تنظيم شناختى هيجان و مهارت هاى مقابلهاى مي باشد.

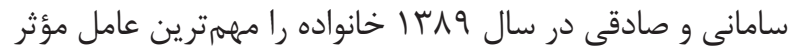

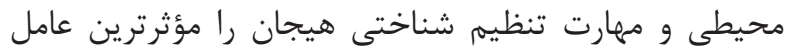

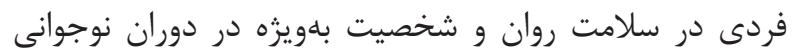

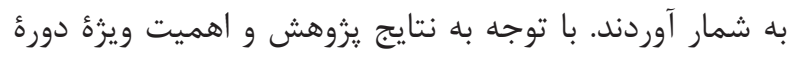

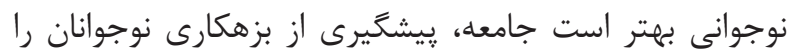

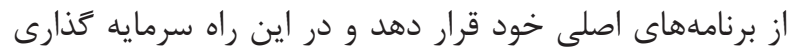

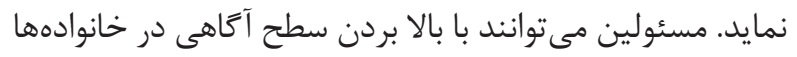

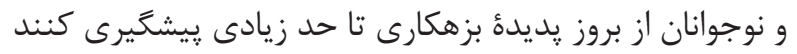

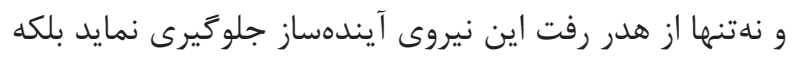
باعث شكوفايى اين افراد و در نهايت رشد رين جامعه شود.

1. Bogaert AF. Personality, delinquency, and sexuality: data from three Canadian samples. Pers Individ Dif. 1993; 15(3): 353-6.

2. Vermeiren R. Psychopathology and delinquency in adolescents: a descriptive and developmental perspective. Clin Psychol Rev. 2003; 23(2): 277318.

3. Navah A, koohpaii M. Effect factor personality on subversion in highschool students in city Ahvaz. Journal of Social Development. 2012; 4:131-143.

4. Berk L. Deveiopement through the lifespan. Thran. Arsbaran Publication. 2001.

5. Cohen MA, Piquero AR. New evidence on the monetary value of saving a high risk youth. J Quant Criminol. 2009; 25(1): 25-49.

6. Khademi A. New approach in criminal behaviors psychoiogy. Tehran. Elm Publication. 2009.

7. Van Giuk E. Adolescent coping theatrical and research perspective. 1st ed. New York. Routledge. 2009.

8. Martins SS, Storr CL, Alexander PK, Chilcoat HD. Adolescent ecstasy and other drug use in the national survey of Parents and youth: The role of sensation-

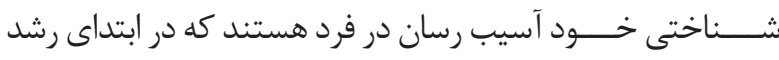

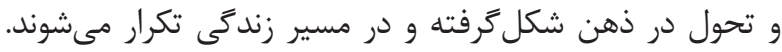

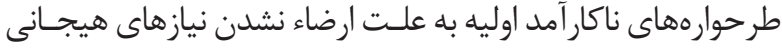

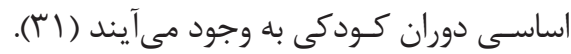

طرحوارههاى ناكارآمد كودكى باعث آسيبذٍ تيرى فرد در جامعه

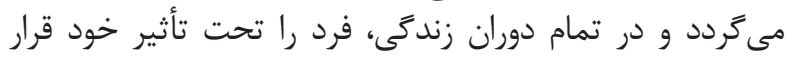

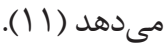

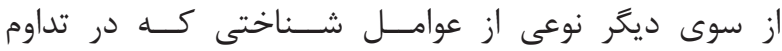

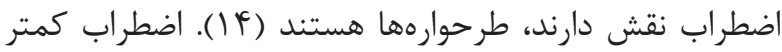

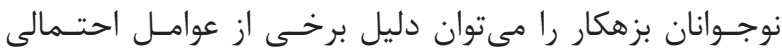

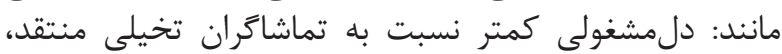

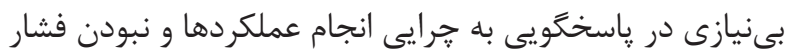

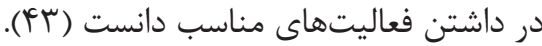

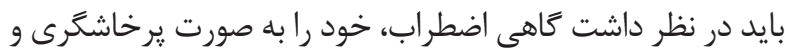

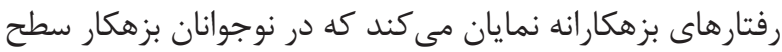

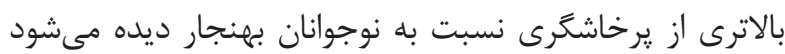

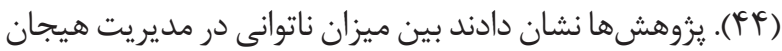

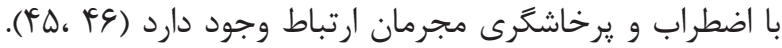

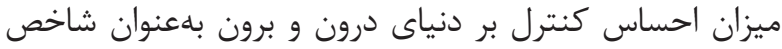

$$
\text { منابع }
$$

seeking, parental monitoring and peers drug use. J Addict Behav. 2008; 33(7): 919-33.

9. Yang J, McCrae R, Costa T, Gao B. Cross-cultural personality assessment in psychiatric populations: the NEO-PI-R in the people's republic of China. J Psychol Assessment. 1998; 11: 359-68.

10. Zhang D, He H. Personality traits and life satisfaction: a Chinese case study. J Social Behavior and Personal. 2010; 38(8): 1112-9.

11. Young J, Klosko J, Weishaar M. Schema therapy: a practitioner's guide. New York. The Guilford Press. 2012.

12. Ruchkin VV, Eisemann M, Hagglof B. Coping styles delinquent of adolescents and controls: the rol of personality and parental rearing. J Youth Adolesc. 1999; 28(6): 705-17.

13. Ghadipasha M, Sardari Poor M, khosh Ravesh S. Comparison of the preliminary discordant schema in psychopath men offenders with personality disorder. Journal of Science Medical. 2014; 19(23): 283-9.

14. Wilkinson PO, Goodyer IM. The effect of cognitive behavior therapy on mood-related ruminative response style in anxiety adolescents. Child Adolesc Psychiatry 
Ment Health. 2008; 2(3): 12-24.

15. Wilson D. Empirical approaches to family assessment. J Fam Ther. 2000; 22(4): 447-9.

16. Erdelja S, Vokal P, Bolfan M, Erdelja SA, Begovac B, Begovac I. Delinquency in incarcerated male adolescents is parent, exposure to more violence at home and at home and in the community, and in the poorer self-image. Croat Med J. 2013; 54: 460-8.

17. Rabi N. Relationship between personality characteristics and rating of the crime of young offenders. Journal of Clinical Psychology Review. 1999; 32: 98-108.

18. Steen N, Janson W. Parental monitoring, personality, and delinquency: further support for a preconception opalization of monitoring. J Res Pers. 2005; 43: 49-59.

19. Afrasiabi M, Akbarzadeh N. A survey and comparison of coping strategies between normal and delinquent adolescents in tehran. Journal of Psychological. 2009; 3: 7- 20.

20. Vermeiren R. Psychopathology and delinquency in adolescents: a descriptive and developmental perspective. Clin Psychol Rev. 2003; 23(2): 277-318.

21. Rio V, Benasive S. Impact of a structured 90-minute play session on development of childern in an orphanage. J Child Health Care. 2009; 28: 95-100.

22. With J. The troubled adolescent. Pergamum Press Publication. 2010; p. 306.

23 Kerr M, Stattin H. What parents know, how they know it, and several forms of adolescent adjustment: Further support for a reinterpretation of monitoring. Dev Psychol. 2000; 36(3), 366-80.

24. Gross JJ. Emotion regulation: affective, cognitive, and social consequences. Psychophysiology. 2002; 39(3): 281-91.

25. Tomson A, Browndfield J. Positive affect facilitates integration of information and decreases anchoring in reasoning among physicians. Organ Behav Hum Decis Process. 1997; 72(1): 117-35.

26. Eetel H. The relation between self-concept and social functioning adolescent. J Adolesc. 2008; 31(1): 1-16.

27. Cichti D. Comparing persistent juvenile delinquents and normative peers with the five-factor model of personality. J Res Pers. 2009; 43(6): 1105-8.
28. Vahedi S, Hashemi T, Einipour J. Investigation of relationship among emotional control, cognitive emotional regulation and obsessive beliefs in high school girl students of Rasht. Adv Cogn Psychol. 2013; 15(2): 63-71.

29. Samani S, Sadeghi L. Examination cognitive emotion regulation questionnaire. Journal of Psychology. 2009; 1: 51-66.

30. Garnefski N, Rieffe C, Jellesma F, Terwogt M, Kraaij V. Cognitive emotion regulation strategies and emotional problems in 9-11-yearold children. Eur Child Adolesc Psychiatry. 2006; 16(1): 1-9.

31. Young J. The relationship between appearance schemas, self-esteem, and non direct aggression among college women. Bachelor of Science in Psychology Cameron University Lawton. 2002.

32. Ahi G. Validity and reliability Young early maladjusted schemas questionnaire. Journal of Psychology and Educative. 2006; 37: 5-20.

33. Panahi shahri H. Validity and reliability speilberger state trait anxiety questionnaire. Journal of Anxiety Disorders. 1998; 12: 18-26.

34. Garnefski N, Kraaij V. Relationships between cognitive emotion regulation strategies and depressive symptoms: a comparative study of five specific samples. Pers Individ Dif. 2006; 40(8): 1659-69.

35. Amin abadi M. Relationship characteristic personality with emotional regulation. Journal of Clinical Psychology. 2009; 4: 70-83.

36. Hasani Z. Examination psychology. Tehran. Behjat Publication. 2011.

37. Young J, Riso H, Dootooeit H, Esteean J. Early maladaptive schema in patients with or without personality disorders: does schema modification predict symptomatic relief. Clin Psychol Psychother. 2005; 12(2): 142-9.

38. Karmi A, Bahrami H, Mohmmadi Aria A, Asghar Nejad A, Fakhri Z. Relationship early maladjusted schemas with antisocial stbstance disorders in weman. Journal of Applied Psychology. 2013; 26: 93-110.

39. Biochman S. Role of genotype in the cycle of violence in maltreated children. Science. 2002; 297: 851-4.

40. Heise D. Expressive order: confirming sentiments in social actions. New York. Springer. 2007. 
41. Van Oudenhoren J, Vander Zee K. Predicting multicultural effectiveness of international student: the multicultural personality questionnaire. Int J Intercult Relat. 2002; 26(6): 677-94.

42. Samani S, Sohrabi R, Mansori F. Emotionality emotion regulation, and performance in middle school children. Journal of Psychology. 2011; 40: 397-416.

43. Puklek M, Videc M. Psychometric properties of the social anxiety scale for adolescents (SASA) and its relation to positive imaginary audience and academic performance in Slovene ado descents. J Studies
Psychologica. 2008; 50: 49-65.

44. White R, Bogaert AF. Personality, delinquency, and sexuality: data from three canadian samples. Pers Individ Dif. 2011; 15: 353-6.

45. Markey C, Markey P, Tinsley B. Personality, puberty and preadolescent girl behaviors: examining the predictive value if the five-factor model of personality. J Res Pers. 2006; 37: 405-19.

46. Ashton M, lee M. Empirical theorical and practical advantages of the HEXACO model of personality structure. Pers Individ Dif. 2009; 42: 151-62. 